\title{
ON SALIÉ'S SUM
}

\section{by L. J. MORDELL $\dagger$}

(Received 2 April, 1971)

Let $p$ be an odd prime and let $f(x)$ be a complex-valued function such that $f(x+p)=f(x)$ for all integers $x$. Write $e(x)=\exp (2 \pi i x / p)$, and define $1 / x$ by $\bar{x}$, where $x \bar{x} \equiv 1(\bmod p)$. We consider the sum

$$
S=\sum_{x=1}^{p-1} f\left(a x+\frac{b}{x}\right)\left(\frac{x}{p}\right) \text { for } a b \neq 0(\bmod p),
$$

where $\left(\frac{x}{p}\right)$ is the Legendre symbol. The sum is zero if $\left(\frac{a b}{p}\right)=-1$, as is clear on replacing $x$ by b/ax. Salié has found a result which can be written in the form

$$
\sum_{x=1}^{p-1} e\left(a x+\frac{b}{x}\right)\left(\frac{x}{p}\right)=i^{(t+p-1))^{2}}\left(\frac{a}{p}\right) p^{ \pm}\{e(h)+e(-h)\},
$$

when $h^{2} \equiv 4 a b(\bmod p)$.

This permits of further applications, as I have shown [1] in a forthcoming paper. Recently, [2] K. S. Williams has found a result which can be written more symmetrically as

$$
\sum_{x=1}^{p-1} f\left(x+\frac{1}{x}\right)\left(\frac{x}{p}\right)=\sum_{x=0}^{p-1} f(x)\left(\frac{x+2}{p}\right)+\sum_{x=0}^{p-1} f(x)\left(\frac{x-2}{p}\right) .
$$

If $h \neq 0(\bmod p)$, on making the substitution

(3) becomes

$$
x \rightarrow x / h, \quad f(x) \rightarrow f(h x)
$$

$$
\sum_{x=1}^{p-1} f\left(x+\frac{h^{2}}{x}\right)\left(\frac{x}{p}\right)=\sum_{x=0}^{p-1} f(x)\left(\frac{x+2 h}{p}\right)+\sum_{x=0}^{p-1} f(x)\left(\frac{x-2 h}{p}\right)
$$

Suppose now that $a$ and $b$ are two integers such that $\left(\frac{a b}{p}\right)=1$. Define a value of $h$ by $h^{2} \equiv a b(\bmod p)$. Replace $x$ by $a x$ on the left-hand side of $(4)$. We then have

$$
\left(\frac{a}{p}\right) \sum_{x=1}^{p-1} f\left(a x+\frac{b}{x}\right)\left(\frac{x}{p}\right)=\sum_{x=0}^{p-1} f(x)\left(\frac{x+2 h}{p}\right)+\sum_{x=0}^{p-1} f(x)\left(\frac{x-2 h}{p}\right)
$$

a result of which Williams informs me he was aware.

Williams's proof of (3) is quite simple, but I give a different one. In (3), corresponding terms of the two series on the right-hand side cancel unless

$$
\left(\frac{x+2}{p}\right)\left(\frac{x-2}{p}\right)=\left(\frac{x^{2}-4}{p}\right)=1 \text {. }
$$

† Professor Mordell died on 12 March, 1972. 
On writing $x^{2}-4 \equiv(x-2 t)^{2}(\bmod p)$, this gives $x \equiv t+1 / t$. The same value for $x$ occurs for two values of $t$ unless $x= \pm 2, t= \pm 1$. Hence

$$
\begin{aligned}
\frac{1}{2} \sum_{x \neq \pm 1} f\left(x+\frac{1}{x}\right)\left(\frac{x}{p}\right)+f(2)\left(\frac{1}{p}\right)+\frac{1}{2} \sum_{x \neq \pm 1} f\left(x+\frac{1}{x}\right)\left(\frac{x}{p}\right)+f(-2)\left(\frac{1}{p}\right) \\
=\sum_{x=0}^{p-1} f(x)\left(\frac{x+2}{p}\right)+\sum_{x=0}^{p-1} f(x)\left(\frac{x-2}{p}\right),
\end{aligned}
$$

which is equivalent to (3).

Now put $f(x)=e(x)$, and replace $h$ by $\frac{1}{2} h$. Then the right-hand side of (5) becomes a gaussian sum; whence

$$
\{e(h)+e(-h)\} \sum_{x=0}^{p-1} e(x)\left(\frac{x}{p}\right)=i^{\left(\frac{1}{2}(p-1)\right)^{2}} p^{\frac{1}{t}}\{e(h)+e(-h)\},
$$

where $h^{2} \equiv 4 a b(\bmod p)$. This gives $(2)$ in a slightly different form from that found by Williams.

\section{REFERENCES}

1. L. J. Mordell, On some exponential sums related to Kloosterman sums, Acta Arithmetica; to appear.

2. K. S. Williams, On Salié's sum, J. Number Theory 3 (1971), 316-317.

ST. JOHN's COLLEGE

CAMBridge 